\title{
Kit didático para ensino de robótica
}

\author{
Educational kit for teaching robotics
}

\author{
- Lucas Kato \\ UFSC, Brasil \\ lucaskkato@gmail.com \\ - Rodrigo Braga \\ UFSC, Brasil \\ rodrigo.braga.ufsc@gmail.com
}

\author{
Ana Veronica Pazmino \\ UFSC, Brasil \\ ana.veronica@ufsc.br
}

\begin{abstract}
The paper presents the kit of a robotic manipulator arm for teaching purposes, as a way to popularize robotics in space science and technology UFSC / Joinville, seeking to reach the public high school, technical and higher. As a result, the paper presents a robotic arm kit of low complexity with four degrees of freedom anthropomorphic type materialized via 3D printer, besides having similar functions to the arms used in industries such as teach function and a proposal to implement rectilinear movements, developed from handlers robot kinematics concepts.
\end{abstract}

Keywords: Robotic Arm, Robotic, Education

\section{Introdução}

Nos últimos anos, com o desenvolvimento tecnológico, redução de custos de componentes eletrônicos e crescimento da mão de obra especializada [1], o campo da robótica vem se popularizando e se difundindo cada vez mais nos países em desenvolvimento, principalmente nas indústrias. Segundo a IFR (International Federation of Robotics) [2], em 2013, cerca de 70.000 unidades de braços robóticos foram vendidos no mundo e, em 2011, 60.000. Como reflexo dessa popularização, muitos grupos de pesquisadores e interessados no assunto começaram a investir nessa área através do uso dos robôs como instrumento de auxílio para suas atividades bem como para o estudo dos componentes mecânicos e eletrônicos, os quais são vistos no ensino técnico e superior.

Nessa perspectiva, o artigo apresenta o projeto que contemplou áreas do Design e Engenharia de Controle e Automação, gerando o protótipo de um kit que pode ser utilizado no ensino da robótica.

\section{Robótica e Educação}

As revistas de tecnologia e ciência, as empresas como a Sony, Honda, e nos últimos anos as revistas de economia como "The financial times" tem publicado artigos sobre o desenvolvimento da nova era da robótica e do aumento da robótica na sociedade como publicado no livro de Martin Ford
"Rise of the robots".

Nesse contexto, a educação deve acompanhar a tendência que mostra o caminho da inovação. Deve seguir o fluxo, pois com a rapidez do surgimento de novas ideias para o uso das tecnologias já existentes e avanços dos mesmos, há a possibilidade de maior acesso à tecnologia robótica nas salas de aula no ensino superior, técnico e básico e, inclusive, para uso domestico.

Além do aspecto tecnológico envolvido na robótica educativa, há a imensa contribuição da psicologia, que com seus fundamentos teóricos possibilitou grandes avanços na tecnologia educacional até chegar à robótica.

\section{Bases da robótica educativa}

Nas décadas de 50 e 60, apesar dos computadores ainda serem um aparato de difićlimo acesso à maioria das pessoas, eles foram o estopim de uma grande mudança na forma do ensino-aprendizagem tradicional. Nesse período a corrente "Behaviorista" estava em alta, cujo fundamento estava estruturado em análises de situações que se podiam apenas observar emedir, sem levar em consideraçãoaspectos da mente ou da consciência. Um dos grandes estudiosos dessa corrente foi Burrhus Skinner, o qual formulou pressupostos no processo de ensino e aprendizagem que geram os seguintes passos: conteúdos a ensinar e aprendizagem gradual com objetivos bem específicos e com grau de dificuldade crescente. Décadas depois, por volta dos anos 80 e 90, estando os computadores 
mais difundidos, foram criados programas mais detalhados que buscavam adaptar-se ao ritmo de aprendizagem do aluno [1]. Assim, as famosas TICs - Tecnologias de Informação e Comunicação - mostraram o seu grande potencial no ensino assistido por computador.

Com a dinâmica que o mundo da informática, mais especificamente com a internet, trouxe, as ideias "behavioristas" começaram a mostrar seus pontos fracos, já que seus fundamentos eram rígidos e consideravam o aluno como um instrumento em que não se levava em consideração os aspectos mentais, tão importantes no processo de aprendizagem. Isso proporcionou uma abertura para uma nova corrente da psicologia, chamada Construtivismo, em que considerava a aprendizagem como um processo ativo, ao contrário de só se obter um conhecimento através de uma leitura ou um discurso de um professor [2], sendo o principal objetivo construir o conhecimento e não, simplesmente, transmiti-lo.

Dessa forma, o foco das atividades educativas parte do princípio de manusear algo concreto com objetivos claros e que despertem o interesse dos alunos em determinado assunto, e isso é o que a robótica educacional em conjunto com as TICs tem como ponto forte, podendo ser utilizadas no ensino de diversos ramos do conhecimento, como também, no desenvolvimento de aptidões dos alunos, como raciocínio lógico, trabalho em equipe, imaginação, criatividade e etc. No entanto, tem como principal fraqueza a necessidade de se ter instrutores capacitados, aptos a utilizar essas tecnologias de forma a sincronizar os conhecimentos que os alunos devem compreender com atividades motivadoras e que façam com que os alunos busquem por si só solucionar os problemas colocados a eles.

Um dos grandes passos dados nesse sentido foi o desenvolvimento da Linguagem LOGO no MIT Massachusetts Institute of Technology - dirigido por Seymour Papert na década de 60 [3]. O Logo é uma linguagem derivada da linguagem de programação Lisp [4]. No início o projeto possuía um robô móvel que podia ser controlado via computador. Mais tarde, esse robô foi substituído por uma tartaruga gráfica, vista na própria interface do programa, podendo ser controlada por crianças devido a sua linguagem de programação ser simples e intuitiva, além disso, devido a sua ampla aplicação em assuntos distintos como matemática, música, robótica, telecomunicações, entre outros, ela foi bem aceita em estudos mais sofisticados. Mais tarde, nos anos 80, foi desenvolvido um sistema com a interface de programação Logo, utilizando motores, sensores e os blocos da famosa marca LEGO no MIT Media Lab. O projeto foi um sucesso entre professores e alunos, iniciando uma nova etapa na robótica educacional.

\section{Contribuições do projeto na robótica educativa}

Com base na evolução de recursos tecnológicos e inovações que contemplam essa modalidade de educação, o projeto do kit apresentado neste artigo buscou criar um produto com as tecnologias mais atuais e difundidas no mercado, sendo sustentado pelas ideias construcionistas, ou seja, aprender fazendo.

Existem diversas áreas de estudo quando se fala em robótica, como a mecânica, eletrônica e computação. Para o ensino em nível técnico o braço robótico deveria auxiliar na introdução à programação, possuindo uma interface com linguagem de programação simples, de alto nível e semelhante às que são utilizadas por alguns modelos reais, como o ACL (Advanced Control Language) e o Scorbot ER-VII [5]. Também o kit buscou aproximar a lógica de movimentação dos modelos industriais, como por exemplo, gravar inúmeras posições do órgão terminal do braço e fazê-lo seguir esses pontos de forma retilínea ou não.

Dessa forma, o objetivo do projeto do kit foi que o programa disponível, ou seja, à programação do microcontrolador utilizado, seja aberto, dessa forma, o usuário poderá alterar as funcionalidades do braço, incentivando-o, inclusive, a construir outros modelos de manipuladores. O que torna interessante no ensino superior em robótica, podendo ser verificado como os parâmetros físicos do braço podem dificultar a programação para a geração de trajetórias com a Cinemática Direta e Inversa.

A implementação da proposta para a movimentação retilínea do efetuador ou órgão terminal do braço robótico necessita de conceitos de cinemática de manipuladores robóticos, tais como movimentos de corpo rígido, cinemática direta e inversa. Estes conceitos são comumente vistos em disciplinas de robótica nos cursos de graduação. Pelo fato de envolverem teorias matemáticas que não são triviais para uma correlação com a prática, muitos estudantes se sentem desmotivados com o assunto, o que poderia ser contornado com a observação e análise de um modelo real de um braço robótico apresentando como ele se comporta de acordo com certos parâmetros dimensionais do robô.

\section{Manipuladores robóticos}

A estrutura mecânica de um braço robótico consiste basicamente de elos (parte rígida que se movimenta de acordo com a junta), juntas (articulação), punho e um órgão terminal, podendo ser de diversos tipos dependendo do que se deseja fazer, como garras, dispositivos de sucção, ponteiras para soldagem e etc. De um modo geral, o braço (elos e juntas) proporciona a mobilidade, o punho, a agilidade e o órgão terminal realiza a tarefa desejada [6]. Na Figura 1é apresentado 
o modelo de um braço com suas partes estruturais. Onde se vê (First arm e second arm), ali estão os elos, e (1st axis, 2nd axis...), as juntas. $O$ punho é composto pelo $4^{\circ}, 5^{\circ}$ e $6^{\circ}$ eixo ou junta.

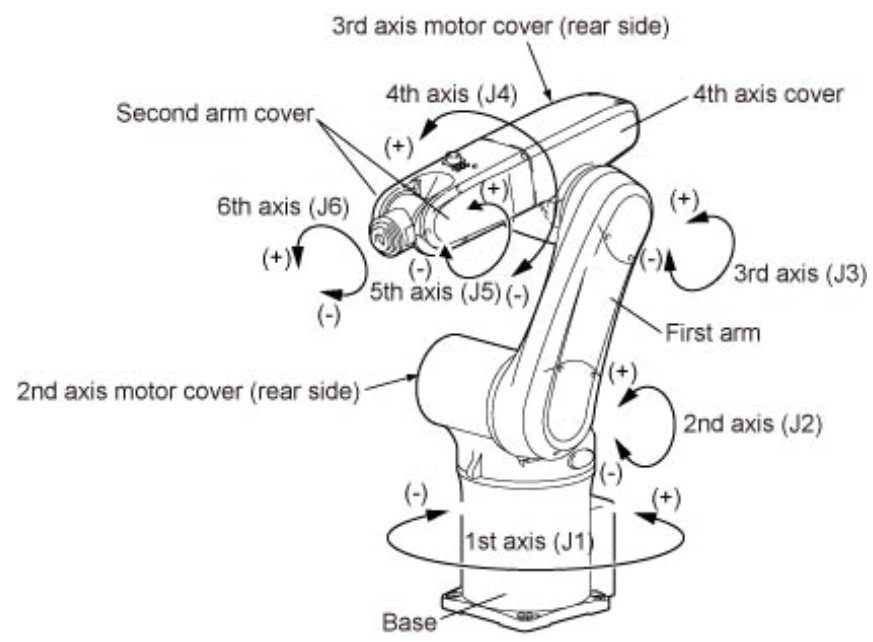

Figura 1: Modelo de braço.

Os braços também podem ser caracterizados como cadeias cinemáticas abertas ou fechadas. As abertas possuem uma única sequência que liga a base do robô até o órgão terminal, já as de cadeia fechada possuem mais de uma possibilidade.

A mobilidade do robô é determinada, principalmente, pelo tipo de junta utilizada, podendo ser rotativa ou prismática. A rotativa permite um movimento rotativo relativo a elos adjacentes à junta, enquanto que a prismática, permite movimentos translacionais. $\mathrm{Na}$ cadeia cinemática aberta, cada junta representa um grau de liberdade (degree of freedom) ou DOF.

\section{Projeto do Kit}

Para o desenvolvimento do projeto do kit foram analisados três kits de robótica, com a possibilidade de se construir diversas estruturas, e três kits com estrutura bem definida - braço robótico.

Os kits analisados foram: Lego Mindstorms EV3 [7]; Linxmotion AL5D [8]; Modelix kit 411[9]; RoboFácil [10]; Hajime [11] e EasyArm DS [12]. A seguir são apresentados os detalhes de cada um.

1. O kit da Lego Mindstorms EV3, é a última geração desse modelo e é mundialmente conhecido devido à sua própria marca e a sua inovação no ramo de brinquedos, chamando a atenção de jovens e adultos interessados no tema da robótica. Ele vem com 601 peças, 4 transdutores, 3 motores, software de programação e inúmeras outras funcionalidades.

Apesar de ele vir com um número bem limitado de atuadores e transdutores, suas peças modulares permitem que se criem uma infinidade de estruturas, inclusive braços robóticos, que a partir de um ambiente de programação visual, semelhante ao LabView ${ }^{1}$, permite-se criar rotinas de movimentações bastante interessantes e até complexas.

Essas características o tornam uma ferramenta que pode ir muito além de um simples brinquedo, sendo inserido nas escolas e universidades como um meio facilitador de aprendizagem.

Como vantagens pode-se citar a facilidade de se programar, já que a linguagem é intuitiva e visual - ícones; possibilidade de se comunicar com outros módulos EV3; além de rodar o programa sem estar conectado a um computador.

Em contrapartida a arquitetura tanto de hardware e software é fechada, o custo é relativamente alto em comparação a outros produtos similares e há uma grande limitação na quantidade de transdutores e motores que podem ser utilizados.

2. O Modelix kit 411dentre os modelos nacionais, pode-se dizer que é um dos maiores concorrentes da Lego Mindstorms devido à grande quantidade de peças, motores e sensores que são disponibilizados, além do software de programação que é bastante intuitivo.

O produto foi desenvolvido com foco nos alunos de ensino fundamental e médio como público alvo, sendo interessante a sua utilização no ensino superior.

Como não há estrutura física pré-definida, considerase esse produto com alta flexibilidade, sendo que suas peças devem ser conectadas com porcas e parafusos, o que torna a sua montagem um pouco mais dificultosa em relação ao kit da Lego descrito anteriormente.

O software de programação possui plataforma fechada. A linguagem utilizada é do tipo visual, baseada em fluxograma, tornando-o bastante interessante para fins didáticos, uma vez que a base da construção de programas ou softwares parte deste método de dispor as informações em um fluxo de dados.

Além disso, o kit vem com um $C D$ que inclui diversos cursos que ensinam a base para se utilizar microcontroladores, aprender alguns conceitos de robótica, e também, manuais para a utilização do software e de modelos de projetos que podem ser feitos com o kit.

Como vantagens, verifica-se: alta flexibilidade, bons recursos de apoio com manuais e cursos, software com linguagem de programação bastante intuitiva, possibilidade de se construir um braço robótico com os recursos disponíveis, entre outros.

Como desvantagem: tanto a plataforma de hardware e de software é fechada, o custo é relativamente alto e o software perde licença de uso após um ano.

3. O kit RoboFácil não é comercializado, no entanto, por ser um projeto resultante de uma dissertação de mestrado possui todo o seu desenvolvimento bem detalhado, sendo possível utilizá-lo como referência para a construção de projetos similares.

1 LabView - Software da empresa National Intruments, muito utilizado na medição ou controle de sistemas eletroeletrônicos através de uma interface de programação visual. 
O kit não vem com atuadores e sensores, apenas com a placa de desenvolvimento (hardware) e um programa baseado em VPL. Nele estão incluídos alguns plugins, que são circuitos externos que permitem a comunicação da placa com o mundo externo. No total são quatro: controle de motores de passo, sensor de luminosidade, sensor de temperatura e LEDs².

Pelo fato do projeto RoboFácil ser um protótipo, sua estrutura física ficou demasiado grande, não sendo interessante para aplicações em robótica embarcada.

Quanto ao software, a programação se assemelha bastante com o programa LabView, em que existem duas interfaces, uma é onde o usuário escolhe e dispõe os dispositivos a serem utilizados, na outra é onde se constrói o fluxo de atividades a serem executadas, tornando-o intuitivo e de fácil programação, porém ele não é open-source ${ }^{3}$.

Esse kit de desenvolvimento tem como vantagem: arquitetura hardware disponível; custo baixo; programação intuitiva e de fácil aprendizado; projeto do produto bem detalhado.

Como desvantagem verifica-se: estrutura física demasiado grande; não contém manual de instrução; não vem com materiais para montar a estrutura física de qualquer outro projeto nem com transdutores e atuadores.

4. Linxmotion AL5D possui uma estrutura rígida sem flexibilidade, com 6 servo motores e 4 graus de liberdade, com a opção de adquiri-lo com ou sem placa de controle da própria empresa, a qual permite o controle de 32 atuadores.

Além disso, a empresa disponibiliza três tipos de softwares que podem ser comprados separadamente: o primeiro, chamado de FlowBotics Studio, utiliza a programação de tipo visual, em que o usuário movimenta um braço virtual, podendo criar rotinas de repetição. O segundo simula um Teach Pendant ${ }^{4}$, comumente usado no meio industrial, sendo possível gravar diversas posições e depois fazer com que o robô percorra a trajetória criada através desses pontos.

As vantagens observadas foram as seguintes: opção de software que permite controlar o robô de forma visual, sendo possível que crianças sem grandes conhecimentos de lógica de programação pudessem controla-lo; opção de software que simula um Teach Pendant, o qual é muito utilizado na indústria, além de ser possível visualizar no programa conceitos relevantes no que tange à robótica, como valores de torque nos atuadores, volume de trabalho, e etc.; robustez física; grande número de portas de saída da placa de controle da empresa, sendo possível utilizar mais de um robô com a mesma placa;

A desvantagem é que a arquitetura de hardware e software é fechada; o circuito eletrônico e os fios são expostos ao ambiente; o custo é relativamente alto e não há flexibilidade para montar outras estruturas.

2 LED - Light Emitting Diode. 3 Open-source - Código aberto.

4 Teach Pendant - Trata-se de um painel de acionamento e controle de robôs.
5. O kit Hajime faz parte de um projeto não comercializado, possuindo sua arquitetura de hardware aberta. Trata-se de um braço robótico com seis juntas rotativas, tendo sua estrutura física construída a partir de materiais sucateados e não utiliza circuitos eletrônicos externos ao computador para comandar os motores. Toda a comunicação é feita via porta paralela.

Para o controle dos motores é utilizado um software com dois níveis de abstração. O primeiro foi planejado para que crianças pudessem manuseá-lo, tendo como linguagem do tipo icônica, em que é possível escolher quatro funções para cada atuador: adicionar, mover, parar ou carregar. $\mathrm{O}$ argumento dessas funções são valores de ângulos.

No segundo também é possível movimentar os atuadores individualmente, sendo que, neste caso, a resolução dos passos do motor é melhor $\left(0,5^{\circ}\right)$. Como opção, ainda é possível controlar o robô via joystick.

Devido à limitação de funcionalidades do software, o mesmo deixa a desejar na questão do aprendizado de conceitos de robótica para jovens do ensino médio e superior, no entanto, o protótipo exige que os alunos tenham um conhecimento básico de eletrônica para poderem utilizar a porta paralela, a qual deve ser conectada com os motores.

Ogrupo desenvolvedor do projeto não divulgou os valores investidos. Segundo Sasahara, as vantagens são: "arquitetura de hardware aberta; design e construção simples; materiais de fácil manuseio e baixo custo; não utiliza circuitos eletrônicos de processamento externo para executar os movimentos". E ainda pode-se dizer que a interface com o usuário é bastante simples, podendo ser utilizada por crianças que não possuem conhecimentos de eletrônica.

As desvantagens observadas são: o software não é open-source; necessita-se de conhecimentos básicos de comunicação via porta paralela; limitação do software quanto às funcionalidades.

6. EasyArm DS não se enquadra como um kit de robótica educacional, mas sua estrutura e componentes permitem a criação de um módulo com fins didáticos.

Sua estrutura geométrica é do tipo articulado ou antropomórfico com quatro graus de liberdade, sendo que os três primeiros motores, partindo da base, servem para posicionar o órgão terminal no espaço 3D e o último, orientar o órgão terminal no espaço tridimensional.

O conjunto vem com dois motores de passo que controlam a posição da base, três servomotores para o controle das outras juntas e a abertura e fechamento do órgão terminal e sensores ópticos para os motores de passo que servem para referenciá-los (set point). Não estão inclusas placas controladoras nem software de programação dos atuadores.

Apesar de se utilizar motores mais robustos na base do robô, tornando-o capaz de levantar cargas mais pesadas em relação aos outros braços vistos anteriormente. $\mathrm{O}$ atuador do órgão terminal é um servomotor comumente utilizado em aeromodelismo, sendo necessário apenas um valor de 
referência - ângulo - para que ele posicione seu eixo, ou seja, no modo como foi implantado, não há controle sobre a pressão exercida sobre um possível objeto que esteja neste órgão, criando uma grande limitação quanto à variação do peso da carga.

As vantagens observadas foram: estrutura física de material resistente, barato e de fácil fabricação; pequeno número de juntas, o que facilita o controle do braço como um todo; custo baixo em relação ao modelo Lynxmotion, sendo que este vem com mais recursos; possibilidade de utilizar diversos tipos de controladores a critério do usuário.

Como desvantagens verificaram-se: modularidade nula; não inclui software para controle dos motores; exigem-se bons conhecimentos em eletrônica e software para utilizá-lo como um kit didático.

Com base nas características de cada modelo, foi necessário compará-los por meio de um quadro contendo alguns atributos com seus respectivos pesos, já que cada um deles tem graus de relevância distintos.

Os seguintes atributos foram comparados:

- Fabricação: podendo ser nacional ou internacional. 0 maior peso vai para os produtos nacionais, já que os mesmos podem ter custo mais baixo e são de grande importância para o desenvolvimento econômico e incentivo à pesquisa do país;

- Material: Plástico (PLA) $)^{5}$ ou alumínio. Apesar de o PLA ser o mais caro, o mesmo possui grande flexibilidade para a criação de peças complexas através da impressão 3D, ficando com o peso intermediário.

- Preço: baixo para valores menores que $\mathrm{R} \$ 500,00$ médio para valores entre $\mathrm{R} \$ 501,00$ e $\mathrm{R} \$ 1500,00$ e alto para valores acima de $\mathrm{R} \$ 1501,00$.

- Arquitetura: aberta ou fechada [13]. Somente foi levada em consideração a arquitetura de hardware, já que não se obtiveram informações sobre as de software para todos os modelos;

- Linguagem de programação: visual - fluxograma, icônica ou convencional. Neste caso considera-se icônica aquela em que não se utiliza fluxogramas e não é convencional - aquelas que necessitam digitar textualmente o comando;

- $\quad$ Estrutura: Modular ou não modular, ou seja, o produto pode assumir várias configurações estruturais - formas [14];

Com esses atributos, montou-se um quadro conceitual que indica os pesos de cada característica de cada atributo, o qual é apresentado em seguida. $O$ valor 3 indica maior importância entre as possibilidades de cada atributo e o 1, o menor. Os produtos que não apresentam alguma informação são representados no quadro com o símbolo "---".

5 PLA: Polylactic acid. Usado em impressoras 3D.
Quadro 1: Atributos de análise

\begin{tabular}{|l|l|l|l|l|}
\cline { 2 - 5 } \multicolumn{1}{c|}{} & \multicolumn{1}{c|}{ Pesos } & \multicolumn{1}{c|}{3} & \multicolumn{1}{c|}{2} & \multicolumn{1}{c|}{1} \\
\hline \multirow{4}{*}{ Gerais } & Fabricação & Nacional & $\begin{array}{l}\text { Não comer- } \\
\text { cializado }\end{array}$ & Importado \\
\cline { 2 - 5 } & Material & MDF & Plástico & Alumínio \\
\cline { 2 - 5 } & Preço & Baixo & Médio & Alto \\
\hline Técnicos & Arquitetura & Aberta & --- & Fechada \\
\cline { 2 - 5 } & Linguagem & $\begin{array}{l}\text { Visual - } \\
\text { Fluxograma }\end{array}$ & Icônica & Convencional \\
\cline { 2 - 5 } & Estrutura & Modular & --- & $\begin{array}{l}\text { Não } \\
\text { modular }\end{array}$ \\
\hline
\end{tabular}

Com base nesse quadro, montou-se outro que relaciona os produtos com os atributos e seus respectivos pesos, os quais indicam o grau de relevância de cada atributo.

Por haver kits com características bem distintas, resolveuse separá-los em kits sem forma definida, que são os da Lego, Modelix e RoboFácil, e kits com forma de braços robóticos.

Na penúltima linha é calculada a soma dos valores para cada produto, considerando os pesos. Para os produtos que não contém informação em determinado atributo, este não é levado em consideração na soma.

Por último é feita uma classificação, em que o melhor é aquele cuja pontuação é maior. $O$ seguinte quadro apresenta essas relações de produtos, atributos e pesos.

Quadro 2: Análise dos produtos similares

\begin{tabular}{|c|c|c|c|c|c|}
\hline & & & s de brac & s robótic & \\
\hline \multirow[t]{4}{*}{ Gerais } & Peso & Nome & Lynxm & Hajime & EasyArm \\
\hline & 1 & Fabricação & 1 & 2 & 3 \\
\hline & 1 & Material & 1 & 2 & 3 \\
\hline & 3 & Preço & 2 & --- & 3 \\
\hline \multirow[t]{4}{*}{ Técnicos } & 3 & Arquitetura & 1 & 3 & --- \\
\hline & 3 & Linguagem & --- & 2 & --- \\
\hline & 2 & Estrutura & 1 & 1 & 1 \\
\hline & - & Soma & 13 & 21 & 17 \\
\hline \multicolumn{3}{|c|}{ Classificação } & $3^{\circ}$ & $1^{\circ}$ & $2^{\circ}$ \\
\hline
\end{tabular}

\begin{tabular}{|c|c|c|c|c|c|}
\hline & \multicolumn{4}{|c|}{ Kits sem forma definida } \\
\hline \multirow[t]{4}{*}{ Gerais } & Peso & Nome & Lego & Modelix & RoboFácil \\
\hline & 1 & Fabricação & 1 & 3 & 2 \\
\hline & 1 & Material & 2 & 1 & --- \\
\hline & 3 & Preço & 1 & 1 & 3 \\
\hline \multirow[t]{4}{*}{ Técnicos } & 3 & Arquitetura & 1 & 1 & 3 \\
\hline & 3 & Linguagem & 2 & 3 & 2 \\
\hline & 2 & Estrutura & 3 & 3 & --- \\
\hline & - & Soma & 21 & 25 & 26 \\
\hline \multicolumn{3}{|c|}{ Classificação } & $3^{\circ}$ & $2^{\circ}$ & $1^{\circ}$ \\
\hline
\end{tabular}


Nessa análise obtiveram-se dois que se destacaram: RoboFácil e Hajime. No projeto RoboFácil, o maior destaque está na disponibilização da arquitetura de hardware, com descrições de como foi implementado o circuito eletrônico principal, os displays, portas paralelas, porta serial, conversores $\mathrm{DAC}^{6}$ e $\mathrm{ADC}^{7}$, plug-ins de controle dos motores de passo, led e sensores.

Em contrapartida, a estrutura possui grandes dimensões comparadas às outras e não possui um modelo de robô como demonstração que possa ser utilizado no sistema.

No Hajime, por se tratar também de um projeto no estado da arte, o usuário deve buscar todo o material para a construção do braço robótico, além de utilizar a porta paralela, que vem entrando em desuso. Esses pontos são os que mais se destacam entre os negativos, porém, como vantagem, não possui circuito eletrônico e a interface é simples e intuitiva, podendo ser utilizada nos níveis de ensino mais básicos.

Com base nas análises e na pesquisa teórica foram estabelecidos os requisitos para o projeto do kit. Vistos no quadro 3.

Quadro 3: Requisitos do kit robótico

\begin{tabular}{|l|l|l|l|}
\hline Requisitos & Material & Quantidade & Classificação \\
\hline Juntas & ABS & 3 & Obrigatório \\
\hline Elos & ABS & 2 & Obrigatório \\
\hline Base & ABS & $13 \times 10 \times 4 \mathrm{~cm}$ & Desejável \\
\hline $\begin{array}{l}\text { Garra (órgão } \\
\text { terminal) }\end{array}$ & ABS & 1 & Obrigatório \\
\hline $\begin{array}{l}\text { Acoplamentos } \\
\text { motor/elo/junta }\end{array}$ & ABS & 5 & Obrigatório \\
\hline $\begin{array}{l}\text { Preço total } \\
\text { desejável }\end{array}$ & & $\mathrm{R} \$ .300,00$ & Desejável \\
\hline $\begin{array}{l}\text { Plataforma de } \\
\text { desenvolvimento }\end{array}$ & & 1 & Obrigatório \\
\hline Leitor cartão SD & & 1 & Obrigatório \\
\hline Servomotor & & 5 & Obrigatório \\
\hline Motor de passo & & 2 & Desejável \\
\hline $\begin{array}{l}\text { Driver motor de } \\
\text { passo }\end{array}$ & & 2 & Desejável \\
\hline $\begin{array}{l}\text { Programa de } \\
\text { escrita e envio } \\
\text { de comandos }\end{array}$ & & 1 & Obrigatório \\
\hline $\begin{array}{l}\text { Programa sim- } \\
\text { ulador de Teach } \\
\text { Pendant }\end{array}$ & & 1 & Desejável \\
\hline
\end{tabular}

A materialização do braço foi dada tanto pelo hardware como pelo software. Foi criada a interface de programação, a qual consiste de: botões para a configuração da porta

6 DAC - Digital to Analog Converter.

7 ADC - Analog to Digital Converter. serial, a qual é conectada à placa de prototipagem, e para a abertura da conexão; um terminal para a inserção de funções que vão para o microcontrolador; um editor de texto para a criação de um código que é gravado no cartão SD; botões para verificação da sintaxe e envio do código para o cartão e um campo para selecionar o programa do cartão e executá-lo.

Primeiramente, para que a conexão seja feita, o Arduino e a interface devem estar configurados na mesma porta serial para o envio e recebimento dos dados, e aquele deve estar conectado via USB com o computador da interface. Feito isso, ao clicar em "open Connection" é chamado o método para "abrir" a porta serial do componente TComPort do Delphi. Assim, o aplicativo está apto para a recepção e envio de dados via terminal ou editor de código.

O terminal é construído com o componente TComTerminal, semelhante ao Hyper Terminal do Windows. Estando a porta aberta, cada carater inserido nele é imediatamente enviado pela porta serial ao microcontrolador, onde será feito o tratamento dos dados recebidos. As mensagens que são recebidas do Arduino também são mostradas nesse terminal.

Os códigos criados neste campo devem iniciar com PROGRAM + [nome do programa] e terminar com END. Para verificar a sintaxe, deve-se clicar no ícone "verificar". Se não houver erros, pode-se enviar o programa para o cartão SD clicando no ícone "Enviar p SD".

Havendo algum programa salvo no cartão, pode-se executá-lo clicando no ícone "Run" com a devida seleção do programa desejado no campo à esquerda desse ícone, chamado "Programs in SD".

A principal função do interpretador é invocar as funções da linguagem utilizada pelo microcontrolador através de funções em ACL, as quais são utilizadas pelo usuário e que contém outras funções não nativas, sendo um ACL adaptado.

Conforme se digita os comandos no terminal da interface, os caracteres vão sendo enviados para o interpretador, o qual deve fazer um tratamento dos dados recebidos para poder identificar as funções e os seus respectivos argumentos quando for o caso, gerando, também, tratamento de exceção quando algo estiver incorreto. As Figuras 2 e 3 mostram as peças do kit e o braço montado. E no site: https://vimeo.com/140209101 ou no qrcode pode ser visto o vídeo.

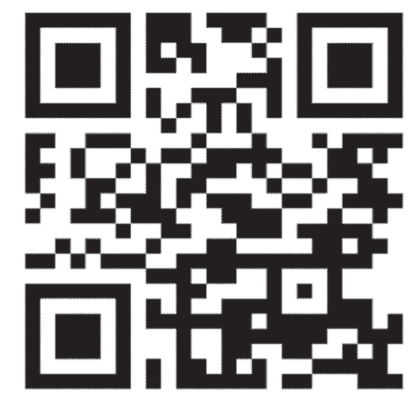




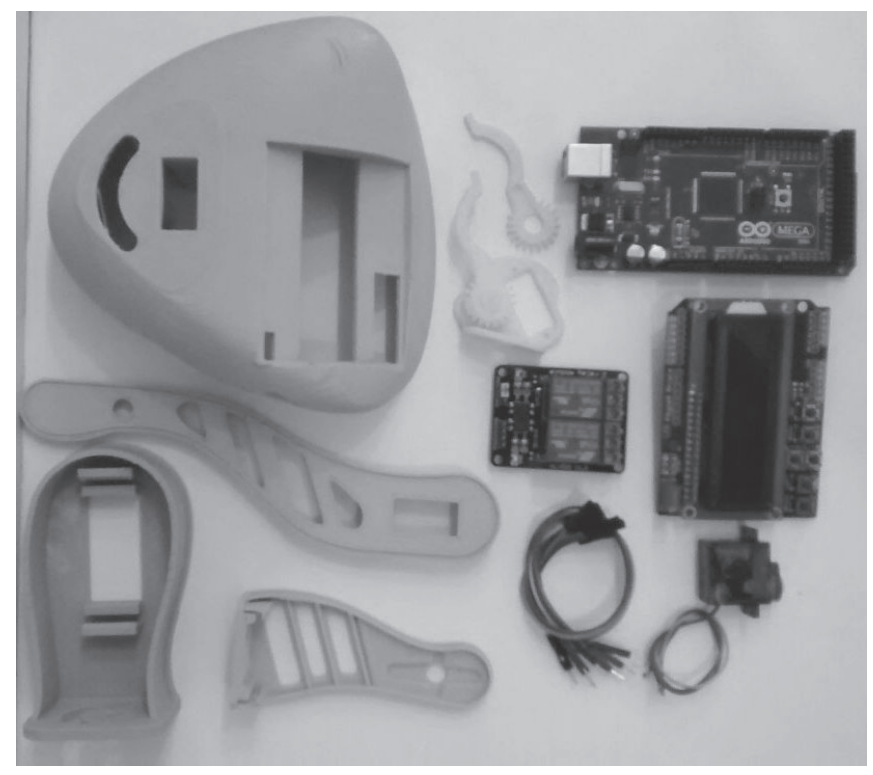

Figura 2: Kit ProntoArm.

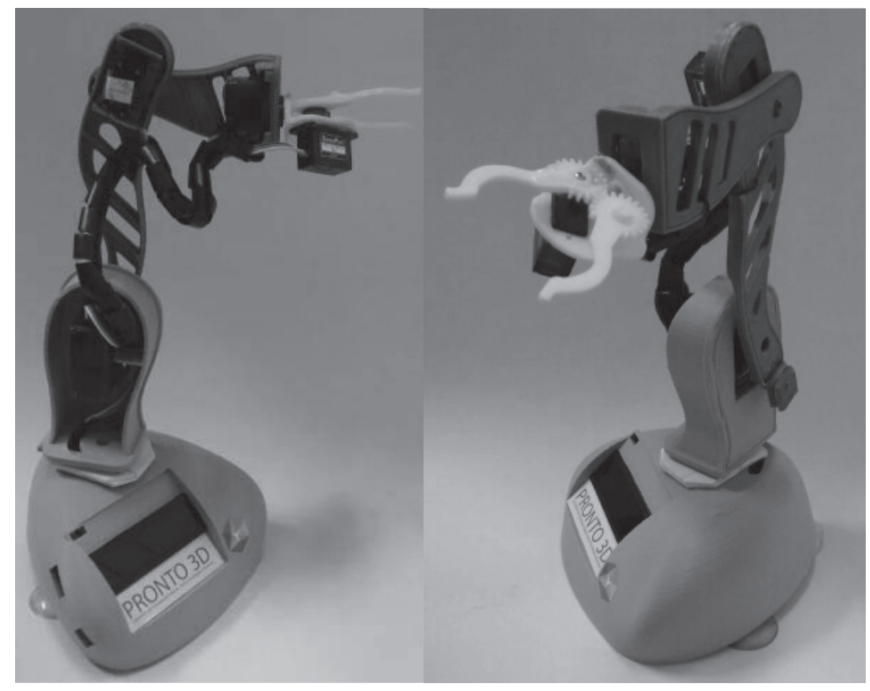

Figura 3: ProntoArm.

\section{Avaliação do kit}

Com base nos dados do produto final, o quadro 4 mostra informações gerais e técnicas no mesmo padrão dos modelos da análise do quadro 2 .

Quadro 4: Informações do kit

\begin{tabular}{|l|}
\hline \multicolumn{1}{|c|}{ Informações gerais } \\
\hline Nome: Kit ProntoArm \\
\hline Fabricação: Pronto3D/UFSC \\
\hline Material: ABS \\
\hline Preço: R\$258,17 (aproximado) \\
\hline \multicolumn{1}{c|}{ Informações técnicas } \\
\hline Arquitetura: Aberta (Software) \\
\hline
\end{tabular}

Linguagem de programação:

ACL modificado

Microcontrolador:

$\mathrm{N}^{\circ}$ de portas (sinal de saída): Depende da placa utilizada.

$\mathrm{N}^{\circ}$ de portas (sinal de entrada): idem

Estrutura: Não Modular

Foram feitos testes do software: ao iniciar o programa da interface, são apresentados ao usuário os arquivos presentes no cartão SD. No caso existem duas formas de se executar o programa do editor, uma é digitando a função run no terminal ou clicando no ícone com o nome desta função. Tanto um como outro invocam a mesma função no firmware. Como mostra a Figura 4.

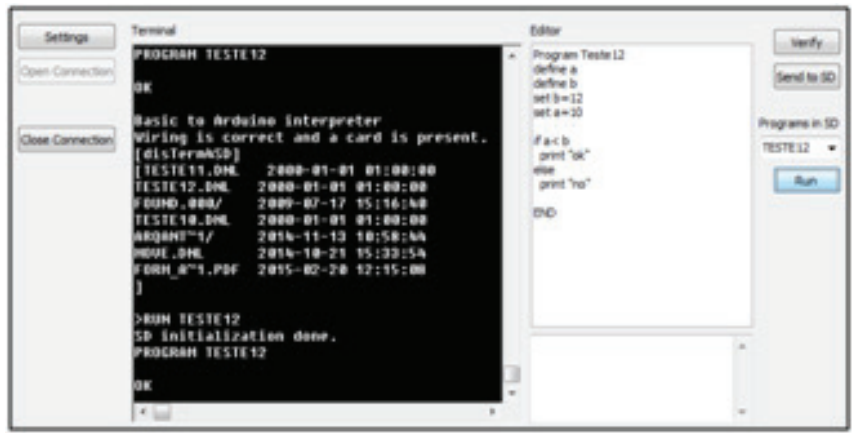

Figura 4: Teste do software.

Posteriormente é feita a movimentação do braço através de comandos via terminal. Iniciando com Manualmode, pressionado Ctrl $+\mathrm{J}$ quando for solicitado para a escolha do sistema de coordenas mais o caractere relativo ao sentido da junta escolhida.

Devido a aspectos estruturais, verificou-se que o braço não é capaz de se movimentar a partir de determinadas posições, porém, com influência externa, o mesmo conclui os movimentos de acordo com as instruções digitadas.

O teste para a movimentação via código no cartão SD também deve iniciar com o procedimento anterior, movendo-o via terminal. Na posição desejada utiliza-se o comando here para criar e gravar as posições de cada eixo em uma variável de posição e se repete esses passos até atingir o número de pontos desejados. Com isso, cria-se o programa no editor. Neste caso, o teste foi feito com a função move a qual move o motor da base em um grau e segue para o seguinte movendo também um grau, e assim sucessivamente até alcançar a posição desejada. O vídeo mostra o movimento do braço.

O resultado mostrou que o Kit ProntoArm possui a melhor classificação levando vantagem sobre os outros por possuir todas as informações do quadro, porém no quesito da linguagem de programação teve a menor nota já que por mais que a linguagem textual seja simples e intuitiva, dificilmente superará uma que também tem essas características e seja visual. Dentro do processo iterativo, a partir da materialização, a análise (teste) o redesign será desenvolver soluções para a 
interface mais intuitiva e tornar a estrutura modular, para permitir maior interação do usuário.

\section{Conclusão}

O kit como mencionado ao longo do artigo buscou contribuir em um campo emergente da grande área da robótica, que se trata da robótica educacional, fundamentada nas teorias de educação provenientes da psicologia, como o construtivismo. Na robótica, buscou-se atuar no campo dos robôs manipuladores, com base nos modelos industriais do tipo antropomórfico.

Para conhecer o que já está presente no mercado e alguns dos trabalhos desenvolvidos no meio acadêmico, foram realizadas pesquisas bibliográficas para a coleta de informações pertinentes para o projeto, formando uma estrutura para a complementação dos requisitos estruturais, funcionais e técnicos.

O kit desenvolvido pode ser dividido em: (1) firmware, (2) interface de programação, (3) estrutura física do braço e (4) componentes de hardware e acionamentos, com um custo baixo ( $\mathrm{R} \$ 258,17)$ em relação aos produtos concorrentes.

No aspecto educacional, com relação ao que foi proposto, os resultados obtidos foram satisfatórios, já que alguns testes - realizados - para as funções do robô mostraram resultados esperados, com exceção da função de geração de trajetória retilínea, porém, no firmware, o qual estará disponível na internet, contém toda a estrutura conceitual para a execução desta função, permitindo o aprendizado básico sobre cinemática de robôs manipuladores.

Salientamos a importância do desenvolvimento de mais pesquisas voltadas a robótica e ao smart design já que a nova onda da TI aponta que no futuro teremos produtos "inteligentes" no ambiente industrial e domestico. Entender seu funcionamento e a necessidade de conhecimento da inter-relação do software e hardware e das diversas áreas de conhecimento envolvidas no projeto de produtos automatizados e inteligentes deve fazer parte do ensino nos diversos níveis de aprendizagem.

\section{Referencias}

[1] Jelden, D.L. (1984). Operationalizing Learner-Crontrolled Education. International Conference on systems Research and Cybernetics, Baden.

[2] Coutinho, C. (2005). Percursos da Investigação em Tecnologia Educativa em Portugal: Uma abordagem temática e metodológica a publicações científicas (1985 - 2000). Dissertação de Doutoramento. Braga: Instituto de Educação e Psicologia da Universidade do Minho.

[3] Papert, S. (1980). Mindstorms: Children, Computers ans Powerful Ideas. New York: Basic Books.

[4] Logo Foundation. (2015, Janeiro). Disponível em: <http:// el.media.mit.edu/logo-foundation/logo/index.html>.

[5] Eshed Robotec. (1998). Scorbot-ER VII Users Manual. 2nd edition. Eshed Robotec.

[6] Siciliano, B.; Sciavicco, L.; Villani, L.; Oriolo, G. (2009). Robotics: Modelling, Planning and Control. 1st edition. Springer Science \& Business Media, $632 \mathrm{p}$.

[7] Lego. (2014, agosto). Disponível em: <http://www.legobrasil. com.br/mindstorms>.

[8] Lynxmotion. (2014, agosto). Disponível em: < http://www. robotshop.com>.

[9] Modelix. (2014, agosto). Disponível em: <http://www. wskits.com.br>.

[10] Miranda, L. C. (2006). RoboFácil: Especificação e Implementação de Artefatos de Hardware e Software de Baixo Custo para um Kit de Robótica Educacional. Dissertação de Mestrado. Rio de Janeiro: IM - NCE - UFRJ.

[11] Sasahara, L. R.; Cruz, S. M. S. (2007). Hajime: Uma Nova Abordagem em Robótica Educacional. Artigo. Rio de Janeiro: Anais do XXVII Congresso da SBC.

[12] EasyArm. (2014, agosto). Disponível em: <http://www. labdegaragem.org/>.

[13] Osier-Mixon, J. M.(2015, janeiro). Hardware Aberto: Como e Quando Funciona. Disponível em: < http://www.ibm.com/ developerworks/br/library/os-openhardware>.

[14] Oliveira, C. B. M. (1999). Estruturação, Identificação e Classificação de produtos em ambientes integrados de manufatura. Dissertação de Mestrado. São Carlos: EESC, USP. 